\title{
Quelques particularités sur les variations de pression dans le clapotis
}

\section{Some remarks on pressure variations in standing waves}

\author{
R. BOUYSSOU \\ PAR \\ ET \\ L. DOUBLET
}

DU LaBORatorre DAUPhINOIS D'HYDRAULIQUE (SOGREAH)

\begin{abstract}
Dans son remarquable traité «Mouvements ondulatoires de la mer», publié en 1944 dans les Annales des Ponts \& Chaussées, M. Miche a établi une théorie de la houle et du clapotis satisfaisant complètement au deuxième ordre d'approximation aux équations de l'hydrodynamique.

En ce qui concerne plus particulièrement les fluctuations de pression dues au clapotis total, M. Miche a mis en évidence l'influence prépondérante des termes du deuxième ordre dans plusieurs cas.

Le présent mémoure rappelle ces particularités curieuses et montre leur confirmation au cours de plusieurs expériences effectuées au Laboratoire Dauphinois d'Hydraulique de la SO.GR.E.A.H.
\end{abstract}

\begin{abstract}
In his noteworthy treatise "Wave motions of the sea", published en 1944 in the "Annales des Ponts et Chanssées", M. Mrche puts forward a theory for waves and slanding waves wich completely satisfies the second order of approximation of hydrodynamic equations.

More particularly with regard to pressure fluctuations due to a total standing wave, M. Miche has shown the preponderent influence of terms of the second order in many cases.

The present article recalls these strange phenomena and shown how they have been confirmed during many experiments carried out at the Laboratoire Dauphinois d'Hydraulique of the SO.CR.E.A.H.
\end{abstract}

\section{AVANT-PROPOS}

Dans son remarquable article « Mouvements ondulatoires de la mer » paru dans les Annales des Ponts et Chaussées en 1944, M. Miche a établi les équations du clapotis irrotationnel satisfaisant complètement au deuxième ordre d'approximation aux équations de l'hydrodynamique.

La loi donnant les variations de pression en fonction du temps fait apparaître quelques particularités soulignées par M. Miche, en particulier les suivantes :

- En profondeur infinie, une fluctuation de pression ayant une fréquence double de celle de la houle incidente;

- Dans presque tous les cas, une dépression maximum supérieure en valeur absolue à la surpression maximum.

M. Mrche a insisté sur ces particularités; il a mis quelquefois l'accent sur les limites possiblẹs de validité de ses formules et enfin il a in- diqué qu'il serait sans doute aisé de vérifier en laboratoire certaines déductions.

L'objet de cette communication est de présenter les résultats obtenus au Laboratoire Dauphinois d'Hydraulique. Nous avons eu l'occasion de faire des enregistrements de la pression due au clapotis, au cours de diverses études expérimentales à deux ou trois dimensions, soit pour le compte du Service d'Etudes pour l'Utilisation des Marées de l'Electricité de France, soit pour la Marine Nationale, mais nous nous limiterons ici à quelques résultats-types obtenus à deux dimensions, ce qui permet une comparaison plus directe avec la théorie.

\section{Installation d'essais}

L'installation d'essais que nous avons utilisée est représentée sur la planche 1 .

Cette installation a été spécialement aménagée par le Laboratoire pour l'étude physique de la houle. Elle a fait l'objet de plusieurs descrip- 


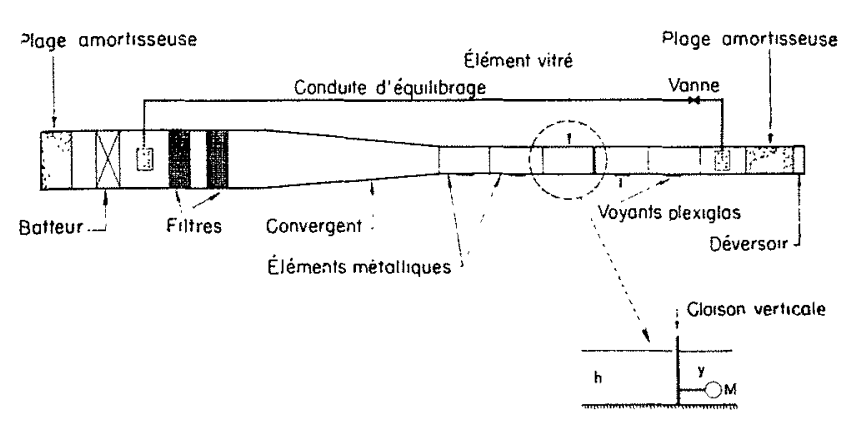

Fis. 1. - Installation expérimentale.

tions dans diverses publications. M. Wallet, notamment, en a présenté une description détaillée dans une communication à la S.H.F. en 1951.

Nous rappellerons simplement que le canal d'essais a une largeur de $35 \mathrm{~cm}$ et une longueur de $14 \mathrm{~m}$. Il est précédé d'un convergent de $11 \mathrm{~m}$ de longueur qui permet la réalisation en régime stable de houles et de clapotis assez cambrés. L'appareil à vagues permet éventuellement de réaliser des trains de houles par fluctuation de la fréquence ou par des variations de l'amplitude du batteur.

Dans le cas particulier des essais qui sont présentés ici, divers clapotis réguliers ont été produits devant une cloison verticale située dans la partie vitrée du canal. La hauteur d'eau a varié entre 20 et $40 \mathrm{~cm}$, la période entre $0,6 \mathrm{~s}$ et $1,2 \mathrm{~s}$ et l'amplitude de la houle incidente entre 4 et $8 \mathrm{~cm}$. Nous avons enregistré les fluctuations de la pression due au clapotis total en un point fixe situé à $5 \mathrm{~cm}$ environ au-dessus du fond du canal.

\section{Appareil enregistreur de la pression}

La planche $\mathrm{n}^{\circ} 2$ donne une vue du microma-

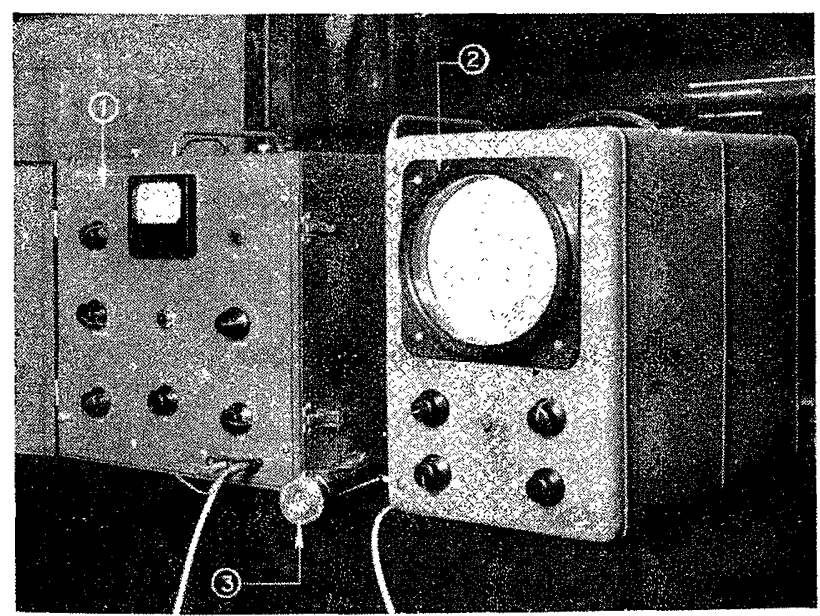

FIg. 2. - Appareil enregistreur pour la mesure des pressions :

1. Bloc électronique,

2. Oscillographe cathodique,

3. Micromanomètre. nomètre à glissement de potentiel mis au point par M. Boudan pour ce genre de mesures, qui demandent une grande précision.

Le capteur présente extérieurement une membrane qui peut être située dans le plan même de la paroi verticale. Sous l'effet des variations de pression, cette membrane se déplace devant une électrode dans un champ électrique constant, ce qui donne lieu à des variations de potentiel entre la membrane et l'électrode. Ces variations de potentiel sont transmises après amplification à un oscillographe cathodique. Il suffit de prendre des photographies du spot qui se déplace par balayage sur l'écran de l'oscillographe pour avoir la courbe de variation de la pression au capteur. Bien entendu, l'échelle de cette courbe est déterminée par un étalonnage préalable en fonction des caractéristiques du clapotis réalisé.

\section{Principales courses théoriques de variation de pression dans un clapotis régulier}

Dans cette partie, nous n'entrerons pas dans le détail des calculs qui ont été effectués pour permettre la comparaison directe de la théorie et des résultats expérimentaux. Ces calculs sont donnés en annexe à la fin du texte. Nous signalerons simplement ici, pour ne pas alourdir cet exposé, la méthode employée dans chaque cas particulier.

Nous rappelons d'abord que la formule générale, qui donne, pour une profondeur finie conslante, les variations de pression dans le clapotis lotal au deuxième ordre d'approximation, a été établie en variables de Lagrange par M. Мiche et publiée dans les Annales des Ponts et Chaussées de 1944. La formule équivalente en variables d'Euler a été donnée par M. BiEsEL dans la Houille Blanche de mai-juin 1952. C'est cette dernière forme que nous avons utilisée puisque nous désirions connaître les variations de la pression en un point fixe de la paroi verticale et comparer ces variations à celles qui sont captées par une prise de pression placée au même point. Nous considérerons ces variations de pression par rapport à la pression statique.

A partir de la formule générale de variation de la pression pendant une période $\mathrm{T}$ du mouvement, qui se réduit à une Ioi sinusoïdale au premier ordre d'approximation, on calcule habituellement le maximum pour $t=\mathrm{T} / 4$ et le minimum pour $t=3 \mathrm{~T} / 4$. L'expression obtenue comprend un terme du premier ordre $\pm 2 a[\operatorname{ch~} m(h-y)] / \mathrm{ch} m h$ et des termes du deuxième ordre, c'est-à-dire faisant intervenir le carré de l'amplitude.

Le terme du premier ordre est habituellement le seul utilisé pour calculer l'amortissement de la pression due au clapotis à diverses profon- 
deurs $y$. Le terme du deuxième ordre donne, par rapport à la pression statique, le décalage de la moyenne des pressions relevées pour la crête et le creux. C'est en quelque sorte l'équivalent de la « surélévation du niveau moyen » pour la surface libre.

Nous allons examiner plusieurs cas typiques en ce qui concerne le décalage de cette pression médiane par rapport à la pression statique et l'allure de la variation complète de la pression pendant une période.

Commençons par les résultats théoriques présentés sur la planche 3 . Nous verrons ensuite les
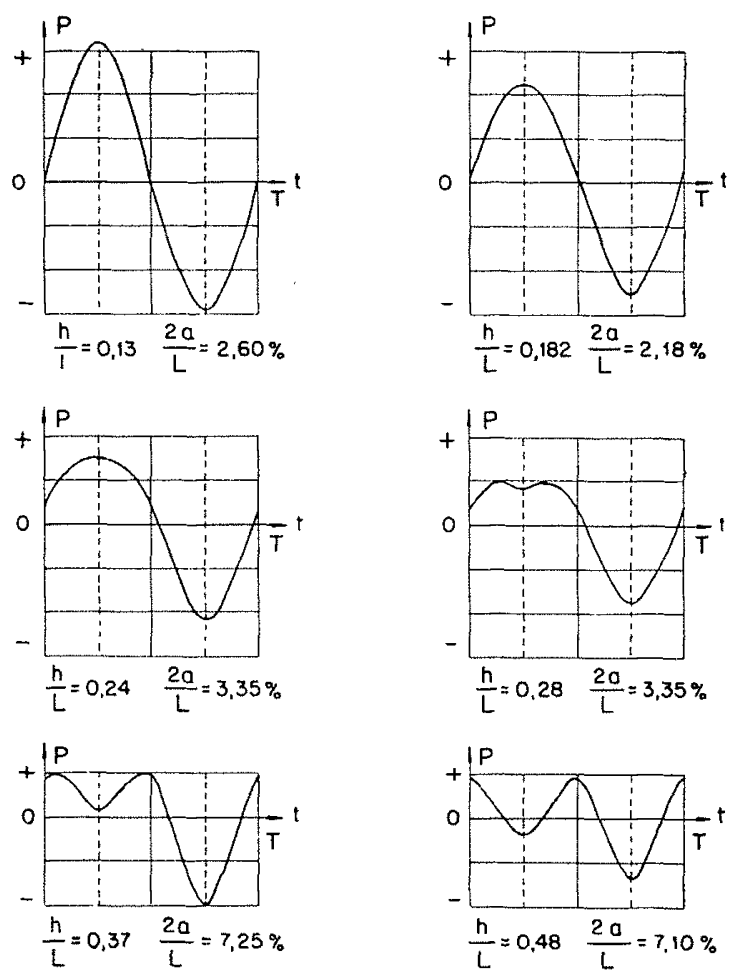

Fia. 3. - Clapotis régulier.

Courbes théoriques $p(t)$.

résultats expérimentaux correspondant aux mêmes clapotis. Nous caractériserons ces clapotis par la cambrure $2 a / \mathrm{L}$ et la profondeur relative $h / \mathrm{L}$ de la houle incidente d'amplitude $2 a$ et de longueur d'onde $\mathrm{L}$ pour la profondeur $h$.

La première courbe théorique de variation de la pression en un point déterminé de la paroi verticale qui produit le clapotis a une allure presque sinusoïdale. La houle incidente a ici une cambrure $2 a / \mathrm{L}=2,6 \%$ et la profondeur relative $h / \mathrm{L}=0,13$. Dans ce cas, la surpression maximum obtenue pour $t=\mathrm{T} / 4$ est supérieure à la valeur absolue de la dépression pour $t=3 \mathrm{~T} / 4$.

C'est l'inverse qui se produit sur la courbe voisine correspondant au clapotis produit par une houle incidente de $2,8 \%$ de cambrure pour une profondeur relative $h / L$ de 0,18 . Ceci correspond au cas le plus fréquent dans la pratique. Il est bien connu en effet que la surpression maximum obtenue en général en profondeur pour un clapotis total est inférieure à la valeur absolue de la dépression maximum. M. MICHE notamment a souligné cette particularité et a suggéré que cela pouvait être une des causes de la chụte côté mer de certaines jetées verticales.

Il est facile de calculer la limite entre les deux exemples ci-dessus $\left|P_{\text {max }}\right|>\left|P_{\text {min }}\right|$ et $\left|\mathrm{P}_{\max }\right|<\left|\mathrm{P}_{\min }\right|$ Ón trouve que cette limite se produit lorsque les termes du deuxième ordre ont une somme nulle dans l'expression du maximum de variation de pression. Cette limite est indépendante de l'amplitude de la houle incidente. Pour un point situé au fond, c'est-à-dire au pied de la paroi verticale, on trouve :

$$
\left|P_{\max }\right|<\left|P_{\min }\right|
$$

pour une profondeur relative :

$$
\frac{h}{\mathrm{~L}}>0,132 \text { ou } \frac{\mathrm{L}}{h}<7,6 \text {. }
$$

C'est par exemple le cas des clapotis produits par des houles de $100 \mathrm{~m}$ de longueur d'onde pour des fonds supérieurs à $13 \mathrm{~m}$. rité.

Passons maintenant à une autre particula-

La courbe médiane de la première colonne de gauche présente au voisinage de $t=\mathrm{T} / 4$ une forme étalée très caractéristique. La houle incidente correspondante a ici les caractéristiques suivantes : $h / \mathrm{L}=0,24$ et $2 a / \mathrm{L}=3,55 \%$.

La courbe de droite est relative à des caractéristiques de la houle incidente assez voisines : $h / \mathrm{L}=0,28, \quad 2 a / \mathrm{L}=3,35 \%$. On voit apparaître un dédoublement du maximum avec un minimum intermédiaire à $t=\mathrm{T} / 4$.

Ceci est dû essentiellement à l'importance des termes du deuxième ordre dans la formule donnant la variation de pression en fonction du temps. Il est facile de déterminer la limite pour laquelle ce dédoublement du maximum se produit et de voir que ceci ne se présente pas au premier ordre d'approximation.

On peut calculer pour cela les valeurs de $t$ pour lesquelles la courbe $p(t)$ présente un maximum. On voit qu'en dehors de $t=\mathrm{T} / 4$ il peut exister d'autres instants définis par une certaine valeur de $\sin 2 \pi t / \mathrm{T}$; il faut $\sin 2 \pi t / \mathrm{T}<1$ pour que ce maximum existe et se situe entre 0 et $\mathrm{T} / 4$.

On' peut encore écrire qu'il se produit à la limite, entre la présence d'un maximum et d'un minimum, un maximum étalé à l'instant $t=\mathrm{T} / 4$, donc que la dérivée seconde est nulle en ce point et on obtient un résultat analogue. 
Cette limite a lieu, par exemple, dans le cas d'un point situé au pied de la paroi verticale, pour un clapotis correspondant à une houle incidente de $100 \mathrm{~m}$ de longueur d'onde, pour une profondeur d'eau de $20 \mathrm{~m}$ et une amplitude de $5,00 \mathrm{~m}$ ou bien avec la même longueur d'onde pour une profondeur de $30 \mathrm{~m}$ et une amplitude de $2,50 \mathrm{~m}$ environ.

Naturellement, ici l'amplitude joue un rôle important, contrairement à ce que nous avons vu pour la limite précédente.

Les courbes inférieures montrent l'importance que peut prendre ensuite cette « double bosse » dans la partie positive de la courbe. Pour la courbe de gauche, les caractéristiques de la houle incidente sont $h / \mathrm{L}=0,37,2 a / \mathrm{L}=7,25 \%$. Pour la courbe de droite, $h / \mathrm{L}=0,48,2 a / \mathrm{L}$ $=7,10 \%$.

Il y a d'ailleurs un cas particulier pour lequel la valeur de la variation de pression $p$ à l'instant $t=\mathrm{T} / 4$ esti nulle et ceci est facile à déterminer dans la formule donnant la valeur de $P_{\max }$ à $\mathrm{T} / 4$ au deuxième ordre d'approximation.

Sur le fond, ceci se produit théoriquement pour le clapotis correspondant à une houle incidente de $100 \mathrm{~m}$ de longueur d'onde par $40 \mathrm{~m}$ de fond si cette houle atteint $5,00 \mathrm{~m}$ d'amplitude.

Pour des clapotis très cambrés et par des profondeurs relatives très grandes, on tend ensuite vers une sorte de dédoublement de la période. Ce sont les fluctuations de pression de fréquence double signalées par M. Mrche qui apparaissent.

Ceci est encore illustré par la planche $n^{\circ} 4$.

Nous avons groupé verticalement les clapotis correspondant à une même cambrure de houle incidente $7,10 \%$ ou $3,55 \%$ et horizontalement
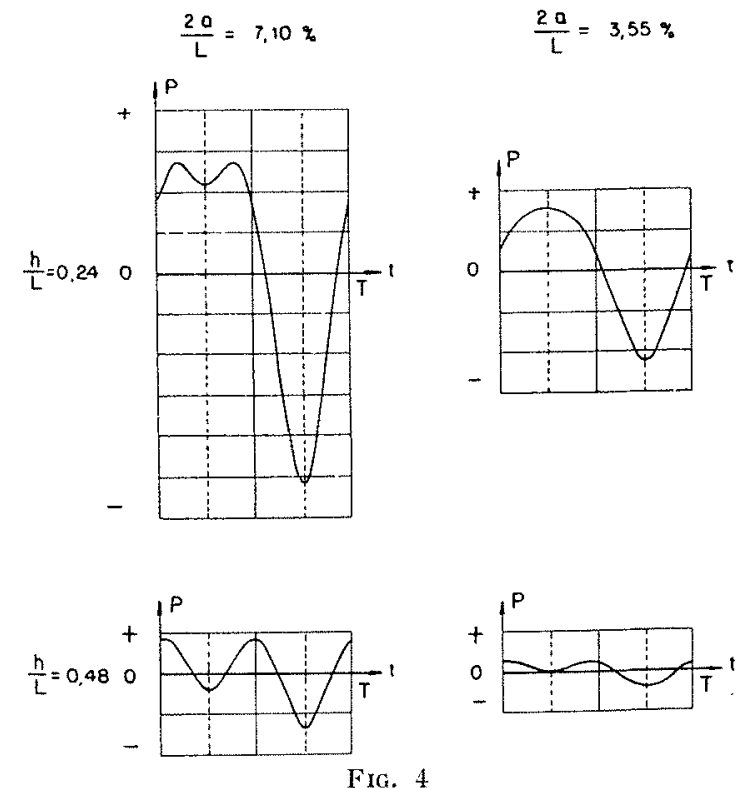

Clapolis regulier. Comparaison des courbes théoriques Influence de $h / \mathrm{L}$ et de $2 a / \mathrm{L}$ ceux qui correspondent à une même profondeur relative 0,24 ou 0,48 .

A partir de la courbe en haut à droite, le fait de doubler $2 a / \mathrm{L}$ et $h / \mathrm{L}$ conduit presque à un dédoublement de la période (courbe gauche inférieure). Les autres courbes sont des cas intermédiaires.

Voyons maintenant ce que donnent les enregistrements de pression pour les mêmes caractéristiques de clapotis.

\section{Résultats expérimentaux}

La planche $n^{\circ} 5$ montre les courbes expérimentales correspondantes. Examinons-les d'abord qualitativement et remarquons leur concordance frappante avec les courbes théoriques.

La première courbe a une allure sinusoïdale et une surpression sensiblement égale à la dépression maximum. Sur la courbe de droite, la dépression maximum se montre en valeur absolue supérieure à la surpression maximum.

Un dédoublement du maximum à $\mathrm{T} / 4$ apparaît dès la troisième courbe et s'accentue sur la quatrième.

Enfin, sur la courbe du bas, une double bosse très caractéristique a été enregistrée. On assiste presque à un' dédoublement de la période sur la dernière courbe.

Signalons en passant que les faibles fluctuations de la pression (de l'ordre de quelques millimètres) ont nécessité dans ce dernier cas l'emploi d'une sensibilité très poussée sur le micromanomètre et ceci a impliqué l'enregistrement simultané des très faibles perturbations parasites qui sont visibles sur l'enregistrement.

Une étude plus détaillée de ces courbes nous a montré que les valeurs relevées pour la dépression maximum correspondaient presque exactement à celles que donne la théorie.

Les valeurs maxima obtenues expérimentalement à $t=\mathrm{T} / 4$ sont en général inférieures de 2 à $5 \%$ aux valeurs théoriques.

De plus, on note expérimentalement l'apparition du dédoublement du maximum un peu plus tôt que ce que donne la théorie. Pour des clapotis très cambrés en grande profondeur, cette double bosse est également plus accentuée sur les résultats expérimentaux que sur les courbes théoriques.

\section{Clapotis irrégulier}

Nous pouvons voir maintenant sur unc autre planche $\left(n^{\circ}\right.$ 6) que les diverses particularités que nous avons rencontrées pour le clapotis régulier peuvent se retrouver tout au moins qualitativement dans le cas d'un clapotis irrégulier.

Indiquons tout de suite que, dans ce cas, la prise de pression n'ćtait pas située au voisinage 


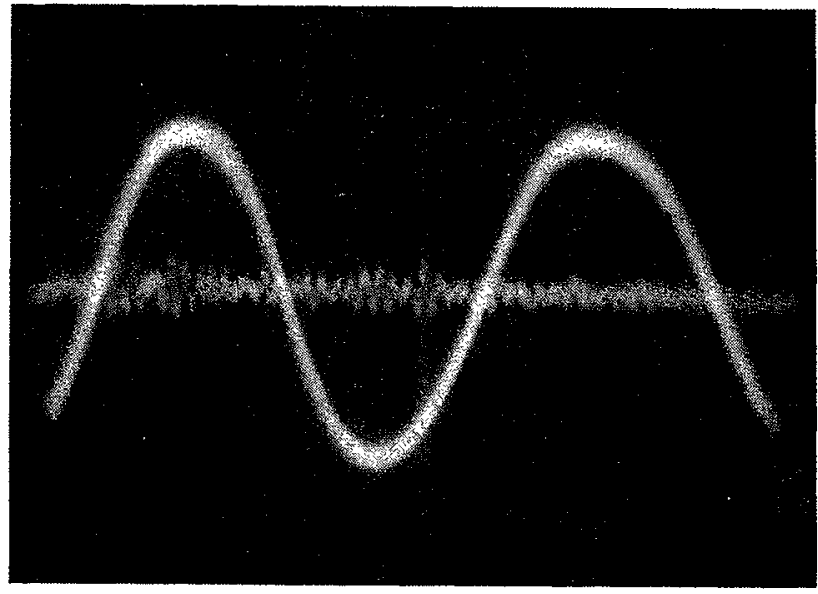

FIG. 5 a

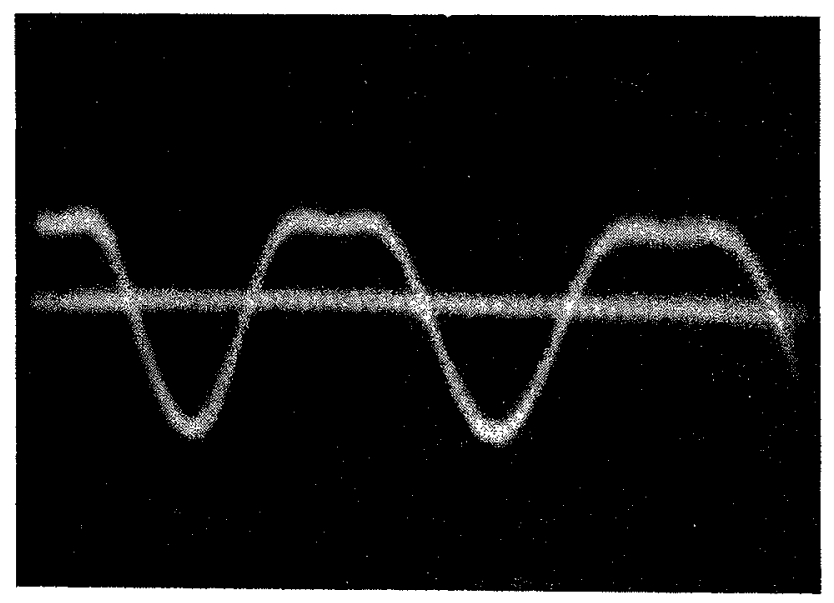

Frg. 5 b

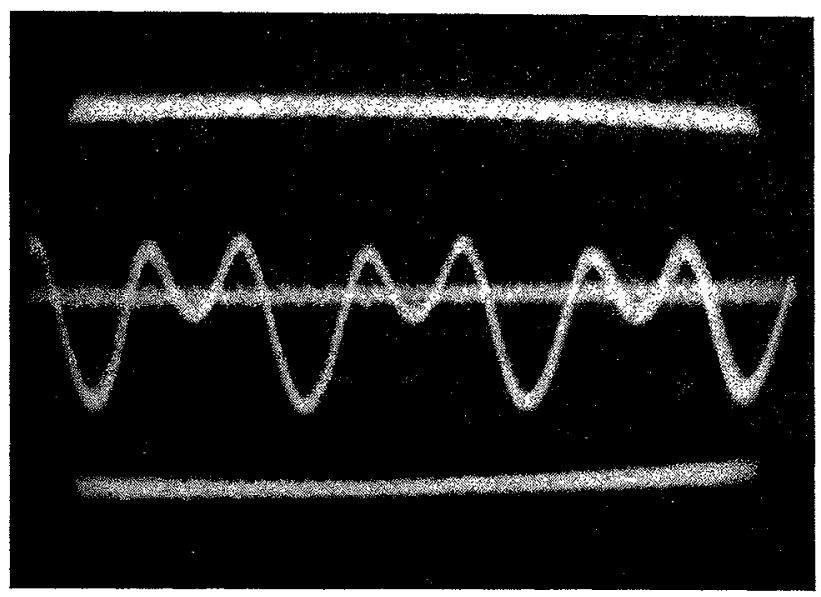

F1G. $5 \mathrm{c}$

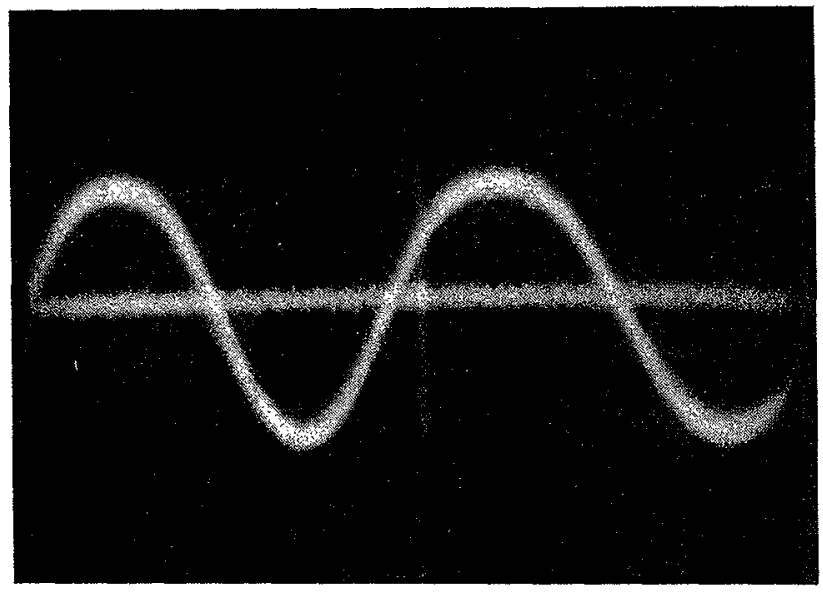

FIG. 5 d

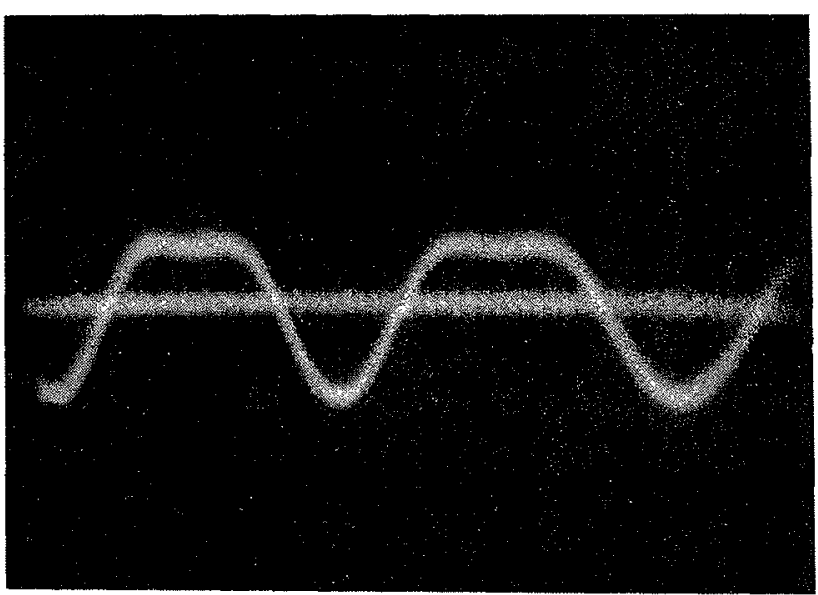

FIG. 5 e

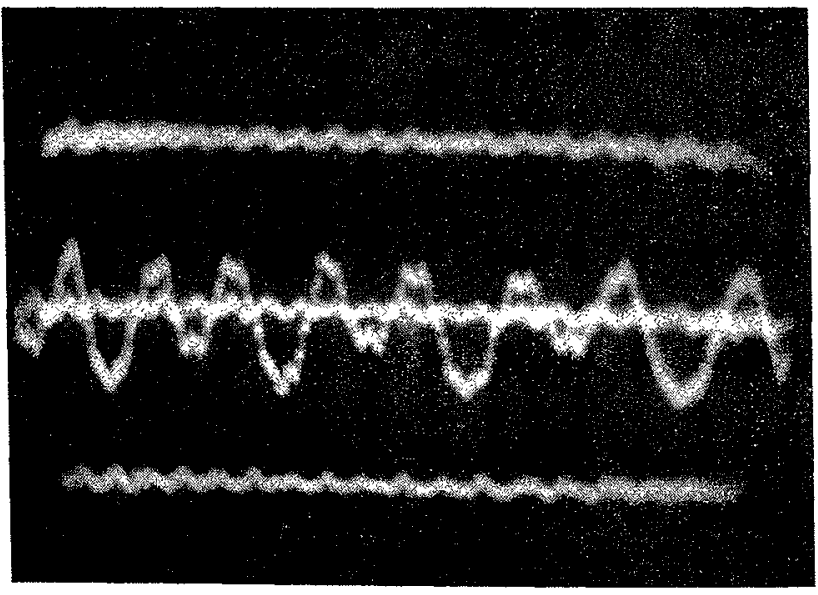

Fig. $5 \mathrm{f}$

FIG. 5. - Clapotis régulier. - Courbes expérimentales $p(t)$. 


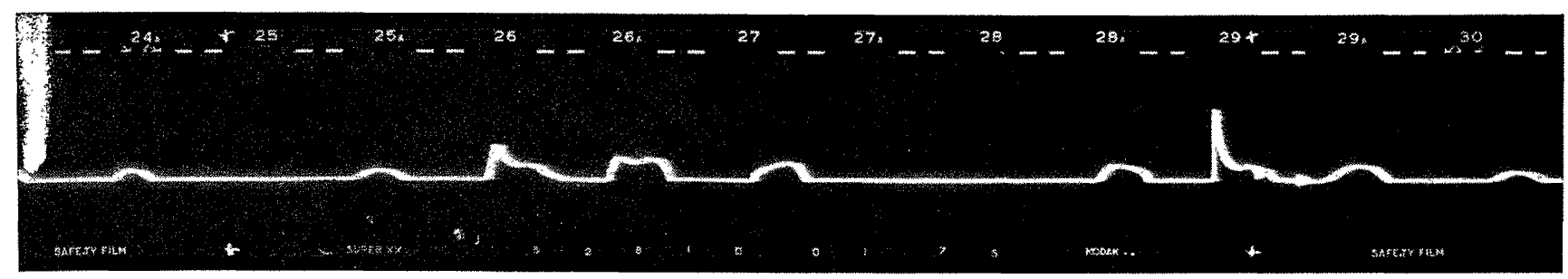

FIG. 6. - Clapotis irrégulier. - Courbe expérimentale $p(t)$ au niveau de repos.

du fond comme précédemment. Elle était calée au niveau de repos. De ce fait, seule la partie positive de la courbe de variation de pression a été enregistrée. L'autre partie est représentée par une horizontale qui correspond à la pression atmosphérique.

On voit sur ce cliché que l'on peut trouver indifféremment une sorte de demi-sinusoïde et une double bosse. On remarque de plus que, dès que cette double bosse devient importante, elle ne reste plus symétrique par rapport au creux intermédiaire. Le premier maximum devient net- tement plus important que le suivant et ceci s'accentue d'autant plus que l'on tend, après le clapotis normal et après le clapotis limite, vers le déferlement sur la paroi verticale avec l'effet de gifle dont on reconnaît une courbe de surpression très caractéristique sur la partie droite de l'enregistrement

Il nous semble donc qu'on retrouve sur ces courbes de pression, entre les phénomènes de clapotis, de clapotis limite et de gifle, une suite normale sur laquelle il était intéressant d'attirer l'attention.

\section{CONCLUSION}

Les enregistrements des variations de pression dans le clapotis que nous venons de présenter montrent, tout au moins pour l'instant dans le cas du clapotis régulier, l'intérêt de la. théorie au deuxième ordre d'approximation.

L'application de cette théorie permet d'ćtablir, pour des points situés au pied de la paroi verticale produisant le clapotis, l'abaque de la planche 7 qui groupe les diverses particularités dont nous avons parlé. Sur cet abaque, la courbe $\mathrm{C}_{1}$ correspondant à une profondeur relative $h / \mathrm{L}$ de 0,13 représente la limite entre le cas où la surpression à l'instant $\mathrm{T} / 4$ est supérieure et le cas où elle est inférieure à la valeur absolue

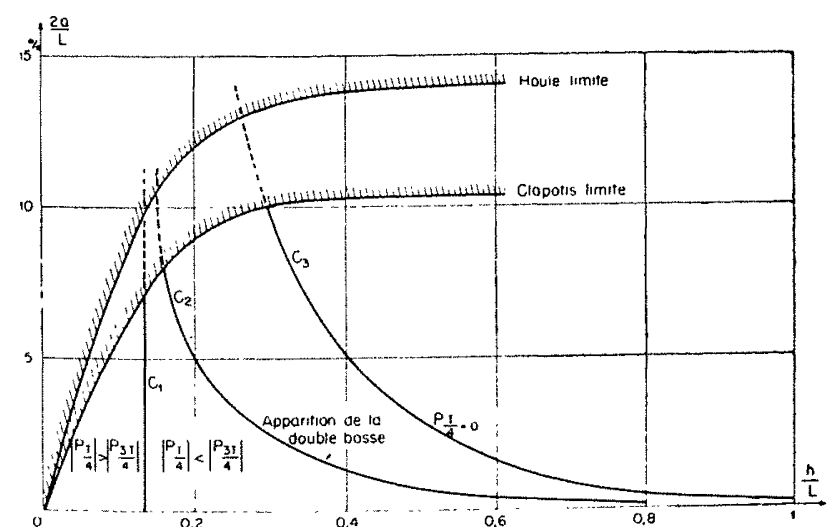

FIa. 7. - Clapotis total. - Variations de la pression au pied de la paroi verticale. de la dépression à l'instant $3 \mathrm{~T} / 4$. Ainsi que nous l'avons indiqué, cette courbe est indépendante de la cambrure $2 a / \mathrm{L}$ de la houle incidente.

La courbe $\mathrm{C}_{2}$ correspond au début de l'apparition théorique de la « double bosse » dans la partie positive de la courbe de variation de pression. Elle est fonction à la fois de $h / L$ et de $2 a / \mathrm{L}$.

La courbe $\mathrm{C}_{3}$, fonction elle aussi de ces deux paramètres sans dimensions, est la limite théorique à partir de laquelle les clapotis très cambrés donnent, pour des grandes profondeurs, une variation de pression négative à l'instant $\mathrm{T} / 4$.

Naturellement, cet abaque doit être limité vers les grandes cambrures non seulement par la courbe correspondant à la houle limite en l'absence de toute réflexion, mais encore par celle qui correspond aux houles de cambrure plus faible qui donnent un clapotis limite par réflexion totale sur une paroi verticale. Nous avons tiré ces deux courbes de la communication de M. Daner à la Société Hydrotechnique de France en 1951 : " Houle limite et clapotis limite. »

Comme on le voit par l'examen de l'abaque et des planches 3 et 5 , nous sommes loin, pour la plupart des clapotis, de ce que donnerait la théorie au premier ordre d'approximation, c'est- 
à-dire à $t=\mathrm{T} / 4$ une surpression toujours positive et égale à la valeur absolue de la dépression. La théorie et l'expérience montrent que, dans certains cas, on a une variation de pression négative à la place du maximum : seul l'écart entre les valeurs à $\mathrm{T} / 4$ et $3 \mathrm{~T} / 4$ reste bon au premier et au deuxième ordre, mais par contre l'écart total ou variation maximum de pression, atteint, du fait des maximums intermédiaires, une valeur plus grande au deuxième ordre et cette valeur est franchie en moins d'une demi-période.

Ces divers résultats soulignent une fois de plus l'intérêt des études expćrimentales et d'un appareillage de mesure très précis pour pouvoir non seulement déterminer les domaines de validité des théories au premier ou au deuxième ordre d'approximation, mais encore dépasser ces domaines de validité comme beaucoup de problèmes le nécessitent.

\section{ANNEXE}

\section{FORMULES EMPLOYÉES}

\section{Notations :}

$h$ : profondeur d'eau au repos;

$\mathrm{L}$ : longueur d'onde de la houle pour la profondeur $h$;

$2 a$ : amplitude de la houle incidente;

$\mathrm{T}$ : période de la houle incidente;

$y$ : profondeur au-dessous du niveau de repos du point considéré de la paroi verticale, produisant le clapotis;

$x$ : section considérée dans le clapotis de longueur d'onde L;

$t$ : instant considéré.

$$
m=\frac{2 \pi}{\mathrm{L}}, \quad k=\frac{2 \pi}{\mathrm{T}}, \quad \gamma=\frac{2 \alpha}{\mathrm{L}}
$$

L'axe $\mathrm{O} x$ coïncide avec la surface de l'eau au repos, l'axe $\mathrm{O} y$ est vertical et dirigé de bas en haut.

Les variations de pression $\mathrm{P}$ en un point à la profondeur $y$ sont considérées par rapport à la pression statique $y$ et exprimées en mètres d'eau pour L, $2 a, h$ et $y$ exprimés en mètres.

Formule générale joe variation de pression DANS LE CLAPOTIS TOTAL (Variables d'Euler, M. Biesel, la Houille Blanche, mai-juin 1952).

$$
\begin{gathered}
\quad p=2 a \frac{\operatorname{ch} m(h-y)}{\operatorname{ch} m h} \sin m x \sin k t \\
-a^{2} m \cdot \frac{\cos ^{2} k t}{\operatorname{sh} m h \operatorname{ch} m h}[\operatorname{ch} 2 m(h-y)+\cos 2 m x-1] \\
+3 a^{2} m \frac{\operatorname{ch} 2 m(h-y)}{2 \operatorname{sh}^{3} m h \operatorname{ch} m h} \cos 2 m x \cos 2 k t \\
\quad+2 a^{2} m \text { th } m h \cos 2 k t
\end{gathered}
$$

En un point situé sur une paroi verticale, c'est-à-dire au ventre du mouvement, on a avec $x=\mathrm{L} / 4$ :

$$
\begin{aligned}
& p=2 a \frac{\operatorname{ch} m(h-y)}{\operatorname{ch} m h} \sin k t \\
& -a^{2} m \frac{\cos ^{2} k t}{\operatorname{sh} m h \operatorname{ch} m h}[\operatorname{ch} 2 m(h-y)-2] \\
& \quad-3 a^{2} m \frac{\operatorname{ch} 2 m(h-y)}{2 \operatorname{sh}^{3} m h \operatorname{ch} m h} \cos 2 k t \\
& \quad+2 a^{2} m \text { th } m h \cos 2 k t
\end{aligned}
$$

Remarquons de suite qu'en profondeur infinie et en des points situés très bas, soit avec $y=h=\infty$, on trouve :

$$
\mathrm{P}=2 a^{2} m \cos 2 k t
$$

c'est-à-dire que les fluctuations de pression ne s'annulent pas par grande profondeur comme dans la houle. Au contraire, la pression subit par rapport à la pression statique des fluctuations de fréquence double de celle du clapotis.

\section{VARIATIONS DE PRESSION POUR $t=\mathrm{T} / 4$ et $t=3 \mathrm{~T} / 4$ :}

Les variations naximum et minimum de pression $\left(\mathrm{P}_{\max }\right.$ et $\left.\mathrm{P}_{\min }\right)$ obtenues habituellement pour $t=\mathrm{T} / 4$ et $t=3 \mathrm{~T} / 4$ sont données par l'expression :

$$
\begin{aligned}
\mathrm{P}_{\min }^{\operatorname{mix}} & = \pm 2 a \frac{\operatorname{ch} m(h-y)}{\operatorname{ch} m h} \\
& +3 a^{2} m \frac{\operatorname{ch~} 2 m(h-y)}{2 \operatorname{sh}^{3} m h \operatorname{ch} m h}-2 a^{2} m \text { th } m h
\end{aligned}
$$

soit en profondeur infinie : $h=\infty$

$$
\mathrm{P}_{\min }^{\max }= \pm 2 a e^{-m y}-2 a^{2} m
$$

soit aussi, en profondeur finie, pour un point situé au pied de la paroi verticale $(y=h)$ :

$$
\begin{aligned}
\mathrm{P}_{\min } \max = \pm 2 a \frac{2 a}{\operatorname{ch} m h}+\frac{3 \alpha^{2} m}{2 \mathrm{sh}^{3} m h \operatorname{ch} m h} & -2 a^{2} m \text { th } m h
\end{aligned}
$$

$\operatorname{Limite}\left|\mathrm{P}_{\max }\right|=\left|\mathbf{P}_{\min }\right|:$ 
L'expression du deuxième ordre s'annule pour :

$$
3 a^{2} m \frac{\operatorname{ch} 2 m(h-y)}{2 \operatorname{sh}^{3} m h \operatorname{ch} m h}=2 a^{2} m \text { th } m h
$$

soit pour :

$$
\operatorname{ch} 2 m(h-y)=4 / 3 \operatorname{sh}^{4} m h
$$

(soit en profondeur finie et au fond pour :

$$
\left.4 / 3 \mathrm{sh}^{4} m h=1, \text { soit } h / \mathrm{L}=0,132\right)
$$

c'est-à-dire pour des caractéristiques de houle indépendantes de l'amplitude $2 a$, mais fonctions de la profondeur relative $h / \mathrm{L}$.

On a alors :

$$
\left|\mathrm{P}_{\max }\right|=\left|\mathrm{P}_{\min }\right|=2 a \frac{\operatorname{ch} m(h-y)}{\operatorname{ch} m h}
$$

ce qui est l'expression bien connue du calcul de la surpression maximum au premier ordre d'approximation.

Signalons qu'il existe un autre cas pour lequel on a : $\left|\mathrm{P}_{\mathrm{T} / 4}\right|=\left|\mathrm{P}_{3 \mathrm{~T} / 4}\right|$ aux instants $\mathrm{T} / 4$ et $3 \mathrm{~T} / 4$ et même $\mathbf{P}_{\max }=\mathrm{P}_{\min }$. C'est lorsque le terme du premier ordre est nul, c'est-à-dire le cas de la profondeur infinie pour les points situés au fond. On a alors :

$$
\mathrm{P}_{\mathrm{T} / 4}=\mathrm{P}_{3 \mathrm{~T} / 4}=-2 a^{2} m
$$

\section{Apparition DE LA « DOUbLE bosse »:}

Les variations maxima de pression peuvent avoir lieu en dehors de T/4 pour des instants $t$ tels que :
- soit en profondeur infinie pour :

$$
\sin k t=-\frac{e^{-m y}}{2 a m\left(e^{-2 m y}-2\right)}
$$

- soit encore en profondeur finie pour les points situés au fond pour :

$$
\sin k t=\frac{\operatorname{sh}^{3} m h}{a m\left(4 \mathrm{sh}^{4} m h+\operatorname{sh}^{2} m h-3\right)}
$$

On a alors $\sin k t=1$ pour :

$$
\gamma=\frac{2 a}{\mathrm{~L}}=\frac{1}{\pi} \cdot \frac{\mathrm{sh}^{3} m h}{\left(4 \operatorname{sh}^{4} m h+\operatorname{sh}^{2} m h-3\right)}
$$

Limite $\mathrm{P}_{\mathrm{T} / 4}=0$ :

Cette limite a lieu pour :

$$
\begin{aligned}
& 2 a \frac{\operatorname{ch} m(h-y)}{\operatorname{ch} m h}=-3 a^{2} m \frac{\operatorname{ch} 2 m(h-y)}{2 \operatorname{sh}^{3} m h \operatorname{ch} m h} \\
& +2 a^{2} m \text { th } m h
\end{aligned}
$$

ou :

$$
a m=\frac{\operatorname{sh}^{3} m h \operatorname{ch} m(h-y)}{\left(\operatorname{sh}^{4} m h-3 / 4 \cdot \operatorname{ch} 2 m(h-y)\right.}
$$

-. soit en profondeur infinie pour :

$$
a m=e^{-m y}
$$

- soit encore en profondeur finie et pour les points situés au fond pour :

$$
a m=\frac{\mathrm{sh}^{3} m h}{\mathrm{sh}^{+} m h-3 / 4}
$$

c'est-ì-dire pour :

$$
\gamma=\frac{2 a}{\mathrm{~L}}=\frac{1}{\pi} \cdot \frac{\operatorname{sh}^{3} m h}{\operatorname{sh}^{4} m h-3 / 4}
$$

$$
\sin k t=-\frac{\frac{\operatorname{ch} m(h-y)}{\operatorname{ch} m h}}{a m\left[\frac{\operatorname{ch} 2 m(h-y)-2}{\operatorname{sh} m h \operatorname{ch} m h}+\frac{3 \operatorname{ch} 2 m(h-y)}{\operatorname{sh}^{3} m h \operatorname{ch} m h}-4 \operatorname{th} m h\right]}
$$

\section{DISCUSSION}

Président: M. Remenmeras

M. le Président se félicite de cette confrontation entre la théorie de M. Mrane et des expériences de laboratoire, confrontation qui confirme les conclusions des travaux de notre éminent collègue.

M. Mrche tient à souligner que les enregistrements fort précis faits au Laboratoire Dauphinois d'Hydraulique sur les variations de pression dans le clapotis, apportent une nouvelle confirmation de l'intérêt de calculs de mouvements ondulatoires au second ordre d'approximation; elle fait suite à celle ressortant des recherches de $M$. Santon sur le profil de la surface libre d'une houle (IV" Journes de l'Hydraulique; question III, rapport $n^{\circ}$ 2). M. SANToN a trouvé que les termes du second ordre rendaient compte de l'observation, mais jusqu'a des cambrures de 2 at $3 \%$ senlement. M. Boysssor montre que les particularites de la variation de pression près du fond sont rendues, à $j \%$ près, pour des clapotis dont l'onde incidente possède des cambrures même exceptionnelles (plus de $7 \%$ ). Ces dcux limites de validité ne sont pas discordantes car, en profondeur, l'approximation réalise par les termes du second ordre est meilleure qu'en surface. 
Par ailleurs, on peut se rendre compte approximativement du pourquoi de l'allure dissymétrique des courbes de surpression et de dépression devant une digue verticale, et du fait qu'il doit s'agir d'un effet du second ordre, bien que fort sensible parfois.

Les pressions extrêmes en pied de la digue, point $P$, sont des moyennes de celles résultant des niveaux d'eau dans les zones les plus proches des maxima ou des minima d'affleurement.
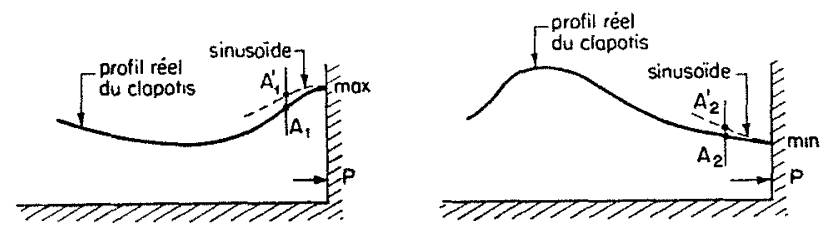

Dans le premier cas, le point $A_{1}$, représentatif de la pression en $P$, est décalé vers le bas par rapport au point $A_{1}^{\prime}$ correspondant à une onde strictement sinusoïdale du premier ordre d'approximation; la surpression en $P$ est donc plus faible que la normale, c'est-à-dire que celle résultant des calculs courants.

Dans le second cas, le point représentatif $A_{2}$ est également plus bas que le point $A_{2}^{\prime}$ correspondant à la sinusoïde habituelle. La pression absolue en $P$ est donc plus faible que celle donnée par la théorie courante; autrement dit, la valeur de la pression par rapport à la pression de l'eau au repos est accrue.

Comme, d'autre part, surpression et dépression sont égales en valeur absolue au premier ordre d'approximation, elles seront inégales si l'on serre la réalité de plus près, la dépression l'emportant sur la surpression.

On voit, en définitive, que cette dissymétrie des pressions résulte de la dissymétrie de l'onde réelle du clapotis telle qu'elle ressort, par exemple, de calculs effectués au second ordre d'approximation, et caractérisée par des crêtes plus aiguës et des creux plus plats.

D'une façon générale, les résultats expérimentaux do M. Bouxssou apportent non seulement une confirmation de vues théoriques, mais ont, de plus, des incidences très appréciables, notamment sur la stabilité des digues verticales.

Il semble, dès maintenant, qu'on puisse délimiter deus domaines dans le calcul approché des houles et des cla- potis : celui dit de première approximation, bien connu et particulièrement simple, utilisable pour des cambrures faibles ou très faibles; celui dit de seconde approximation, qui paraît serrer la réalité de beaucoup plus près dans un domaine couvrant presque la totalité des cambrures courantes.

C'est une conclusion intéressante qu'on n'aurait pu énoncer sans incertitude, avant les contrôles récents et précis effectués, entre autres, par MM. Santon et Bouyssou.

M. de Rouville souligne, à la suite de la communication de M. Bourssou, le relèvement, beaucoup plus considérable que ne l'indiquent la théorie ou les formules, de la surélévation des lames de clapotis au-dessus de l'affleurement maximum théorique - et cela, sans augmentation corrélative de la pression.

Ce fait a été indiqué au cours de plusieurs congrès internationaux de navigation.

On l'a attribué tour à tour :

- soit à l'interférence des lames aux flancs de la baie au fond de laquelle se trouve le port;

- soit à l'action d'un vent local.

Mais on commence à se demander s'il n'y a pas à attribuer un effet au soubassement en enrochements que couronne la muraille verticale de la digue, bien qu'il soit normalement trop immergé pour agir.

A Naples, en particulier, il y a une forte substructure d'enrochements, et la jetée est comme perchée à son sommet. Heureusement, la mer n'y est pas très dure.

II n'en serait pas de même à Douvres, où la partic verticale jaillit du sol même.

Il serait intéressant pour M. Bouyssou d'avoir connaissance du procès-verbal de la dernière réunion du Comité des Efforts des Lames. M. de Rouville le lui fera parvenir dès qu'il sera prêt.

En revanche, il demande à M. Bouxssou de fournir en un nombre suffisant d'exemplaires, sa communication d'aujourd'hui, avec des planches jointes, pour que les membres du Comité des Efforts des Lames en aient connaissance en même temps que du procès-verbal sus-visé.

M. le Président souligne l'intérêt de ces compléments a la communication de $M$. Bouxssou et remercie à la fois son auteur et les personnalités qui sont intervenues dans la discussion.

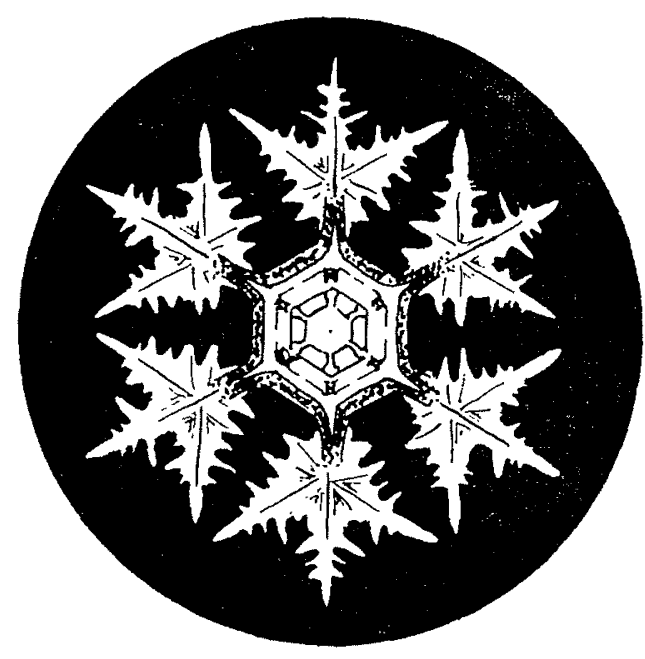

